\title{
Central precocious puberty in a 3 year-old girl with Phenylketonuria: a rare association?
}

\author{
Laura Lucaccioni ${ }^{1,3}$, Bernd C Schwahn ${ }^{2}$, Malcolm Donaldson ${ }^{3}$ and Claudio Giacomozzi ${ }^{3,4^{*}}$
}

\begin{abstract}
Background: Central precocious puberty (CPP) and phenylketonuria (PKU) are two rare conditions, the latter being the rarer. To date, only one case featuring both these conditions has been reported, and hyperphenylalaninemia was assumed triggering CPP.

Case presentation: We present a 3.2 years old girl referred with a 12 months history of breast and pubic hair development, and vaginal discharge. Hyperphenylalaninemia had been identified by newborn screening and PKU subsequently confirmed by plasma amino acid and genetic analysis. Early dietary control of plasma phenylalanine had been excellent afterwards, resulting in phenylalanine concentrations consistently within the recommended range. Clinical scenario, hormonal assessment and imaging were in keeping with true idiopathic central precocious puberty. Treatment with long lasting gonadotropin-releasing hormone analogue led to regression of secondary sexual characteristics.
\end{abstract}

Conclusion: We describe for the first time CPP in a girl affected with PKU but with persistently well controlled blood phenylalanine concentrations. This finding is in contrast to a previous report which suggested persistently high phenylalaninemia levels as potential trigger for CPP in PKU patients. Our report, together with the lack of evidence in published cohort studies of children with PKU, strongly suggests this rare association is coincidental and independent of the presence of severe hyperphenylalaninemia.

Keywords: Precocious puberty, Phenylketonuria, Hyperphenylalaninaemia, Gonadotropin-realising hormone agonist treatment

\section{Background}

True Central Precocious puberty (TCPP), also called GnRH-dependent precocious puberty, can be defined as true puberty, mediated by the hypothalamus, with the onset of secondary sexual characteristics before the age of eight in girls and nine in boys [1-4]. The estimated incidence of TCPP is 1:5.000-10.000 throughout the world [2] with a female:male ratio of between 3:1 and 23:1 [3]. It is less common than, and should be differentiated from, other forms of sexual precocity such as premature thelarche and exaggerated adrenarche. Early activation of the hypothalamic-pituitary-gonadal axis (HPGA) in TCPP is mostly idiopathic, especially in girls [1-4]. However TCPP

\footnotetext{
* Correspondence: dr.giacomozzi@gmail.com

${ }^{3}$ Paediatric Endocrinology Unit, Royal Hospital for Sick Children, Dalnair St, Yorkhill, G3 8SJ Glasgow, UK

${ }^{4}$ Dipartimento di Medicina Pediatrica, Bambino Gesù Children's Hospital, Piazza S. Onofiro 4, 00165 Rome, Italy

Full list of author information is available at the end of the article
}

may be secondarily related to brain tumors (hamartoma especially), brain infections, congenital brain defects, cranial irradiation, insults and injuries to the brain or spinal cord (including cerebral palsy, hydrocephalus and brain ischemia) [4]. Moreover TCPP has been widely described in association with learning disability syndromes, including Angelman, Kabuki make-up syndrome and other genetic conditions in which mental retardation is a key clinical feature [5-8]. Phenylketonuria (PKU) is an autosomal recessive disease caused by a deficient activity of Phenylalanine Hydroxylase (PAH), the enzyme which metabolizes Phenylalanine (Phe) to Tyrosine (Tyr). Deficiency of PAH results in tyrosine deficiency, hyperphenylalaninemia (HP) and accumulation of Phe in other body fluids [9]. If left untreated or not appropriately controlled, patients may develop mental retardation, microcephaly and epilepsy due to the high sensitivity of the immature brain to pathological Phe concentrations. Insufficiently treated patients can also have behavioural problems and some 
may suffer from psychiatric illnesses [10]. To our knowledge only one girl affected by HP has been described in the medical literature with associated TCPP [11]. In their paper the authors highlight the poor control of serum Phe level due to their patient's inadequate adherence to the low Phe diet and hypothesized that chronically elevated Phe concentrations were the trigger of TCPP. Since then, no other data supporting or rejecting this conjecture have been reported. We present the case of a three-year-old girl affected by severe PAH deficiency who was diagnosed with TCPP, and have compared her to the single case previously reported, pointing out differences and similarities.

\section{Case presentation}

A girl was referred to our endocrinology outpatient clinic at 3.2 years with a one year history of increasing breast enlargement followed by the development of pubic hair, body odour and brown vaginal discharge. She had been born at 38 weeks gestation by caesarean section due to maternal pre-eclampsia, birth weight $2.7 \mathrm{~kg}$ $(-0.72 \mathrm{SD})$. Her family history was negative for developmental anomalies. Newborn screening revealed HP with a Phe level of $435 \mu \mathrm{mol} / \mathrm{L}$ on day 5 and she was started on dietary treatment from day 9 of life once severe isolated hyperphenylalaninaemia had been confirmed with plasma amino acid analysis $(975 \mu \mathrm{mol} / \mathrm{L})$. Bi-directional sequence analysis of the PAH gene identified two heterozygote mutations: p.Phe39Leu which is associated with a variably severe phenotype, as well as a second wellcharacterised null mutation, p.Pro281Leu. Her phenylalanine tolerance remained around $200 \mathrm{mg}$ per day throughout the first 2.5 years of life. She showed normal neurodevelopment but for transitory mild speech delay. From infancy to the time of referral the girl underwent weekly Phe measurements with median (range) values of $194 \mu \mathrm{mol} / \mathrm{L}(63-527)$ and thus almost exclusively within the target range of 120-360 micromol/l for children from 0 to 5 years of age in the UK (Figure 1).
On examination in the endocrine clinic the girl was tall in comparison to her parents' height, 0.8 SDS according to normal reference [12], while her mid parental height $(\mathrm{MPH})$ was $-1.2 \mathrm{SDS}$, with a height corrected for MPH of 2.0 SDS. Pubertal staging according to Tanner's method [13] was assessed at B4, P2, A1.

\section{Results}

Bone age was advanced by over one year at 4.62 years using the TW2 (RUS) method [14] leading to a predicted final height below the cut-off of -2.5 SDS. An LHRH stimulation test was arranged and showed a pattern which was diagnostic for TCPP (Table 1). Pelvic ultrasound scan showed a bulky uterus of $4.75 \mathrm{~cm}$ length with a fundocervical-ratio of 1.17 and endometrial thickness of $5.7 \mathrm{~mm}$. Ovaries were 1.70 and $0.78 \mathrm{ml}$ in volume, both within the normal volume range for age although large follicles were evident. Brain and pituitary MRI showed a normal pituitary gland with a mild convexity presentation compatible with the HPGA activation, but no other organic causes for TCPP were detected. Treatment with the GnRH agonist (Triptorelin) was started at the dosage of $11.25 \mathrm{mg}$ every twelve weeks. After nine months, because of persistent breast enlargement and increased height velocity, the interval between injections was reduced to ten weeks. Subsequently the girl showed regression of breast development and a decrease in height velocity. Now aged 4.6 years the patient remains on treatment, showing reduced height velocity and regression of secondary sexual characteristics (Table 1). Throughout treatment her dietary management had been adjusted. Phenylalanine tolerance remains elevated at around $350 \mathrm{mg}$ per day on $\mathrm{GnRH}$ agonist treatment and Phe concentrations have been maintained within the target range with median (range) values of $194 \mu \mathrm{mol} / \mathrm{L}$ (63-527).

\section{Conclusions}

Phenylketonuria was first described by Asbjørn Følling in 1934 as one of the most common inherited metabolic

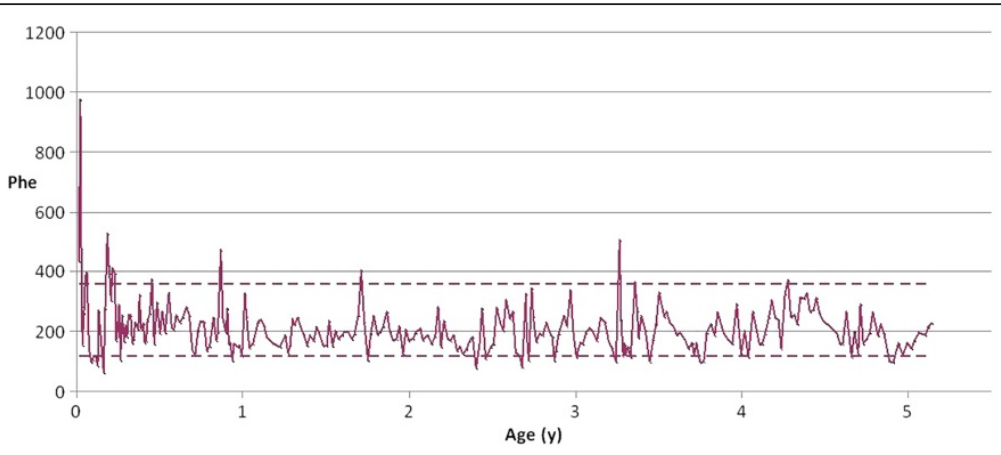

Figure 1 Patient serum Phe concentration from birth. The area within dotted line represents the treatment target range. Phe concentration is in $\mu \mathrm{mol} / /$. 
Table 1 Clinical and biochemical features in a girl with phenylketonuria presenting with sexual precocity aged 3 years

\begin{tabular}{|c|c|c|c|c|}
\hline Age & $3.2 \mathrm{yr}$ & $3.9 \mathrm{yr}$ & $4.2 \mathrm{yr}$ & $4.6 \mathrm{yr}$ \\
\hline Height, cm & $98.8(0.8)$ & $107.5(1.5)$ & $109.7(1.4)$ & $111(1.2)$ \\
\hline Weight, Kg & $16.1(0.8)$ & $18.54(1.1)$ & $18.76(0.9)$ & $19.44(0.8)$ \\
\hline $\mathrm{BMI}, \mathrm{Kg} / \mathrm{m}^{2}$ & $16.43(0.4)$ & $16.0(0.2)$ & $15.6(0.0)$ & $15.7(0.1)$ \\
\hline $\mathrm{MPH}, \mathrm{cm}$ & $156.0(-1.2)$ & & & \\
\hline Ht corrected for MPH, SDS & 2.0 & 2.7 & 2.6 & 2.4 \\
\hline Bone age, yr & 4.62 & - & 5.37 & - \\
\hline $\mathrm{Ht}$ velocity, cm/y & $14.3(5.3)$ & $10.6(2.9)$ & $7.1(0.1)$ & $4.3(-2.2)$ \\
\hline Pubertal status & $\mathrm{B}_{4}, \mathrm{P}_{2}, \mathrm{~A}_{1}$ & $\mathrm{~B}_{2}, \mathrm{P}_{2}, \mathrm{~A}_{1}$ & $\mathrm{~B}_{1-2}, \mathrm{P}_{1}, \mathrm{~A}_{1}$ & $\mathrm{~B}_{1-2}, \mathrm{P}_{1}, \mathrm{~A}_{1}$ \\
\hline Basal/Peak LH, U/I & $1.9 / 33.1$ & & & \\
\hline Basal/Peak FSH, U/I & $2.4 / 6.5$ & & & \\
\hline Triptorelin dose & $11.25 \mathrm{mg} / 12$ weeks & 11.25 mg/10 weeks & 11.25 mg/10 weeks & $11.25 \mathrm{mg} / 10$ weeks \\
\hline
\end{tabular}

SDS is presented in brackets. Height velocity assessed according to Tanner et al. [13].

disorders. The overall incidence of PKU in Europe and North America is 1:10.000-15.000 live births [15]. It occurs, however, more frequently in certain areas such as Turkey (1:3.500-1:5.000), Ireland (1:4.500) or the West of Scotland (1:7.500) [16]. Patients can be classified on the basis of residual enzyme activity as judged by peak blood Phe levels or, better, long-term phenylalanine tolerance. Phe-restricted diets with amino acid and micronutrient supplementation are highly effective in preventing irreversible brain damage and allow for a normal physical and cognitive development [10]. The recommendations for acceptable safe blood Phe concentrations are to some extent age specific [17]. Our patient has a severe enzyme deficiency with a low phenylalanine tolerance, increasing the risk for neurocognitive anomalies. Her parents achieved an excellent dietary control of Phe concentrations with subsequent normal neurodevelopment. To our knowledge, there is only one previous case reported in the literature [11] describing TCPP in a Turkish girl aged 7.5 years with severe PAH deficiency. While most countries represented in the medical literature have universal neonatal screening programs in place to avoid symptomatic PKU, classical PKU was diagnosed late in this patient at 2.5 years of age, indicating that she had been exposed to elevated Phe concentrations from birth. Moreover, satisfactory Phe concentrations could not be achieved in the girl because of poor adherence to dietary recommendations. The authors assumed that persistent poor dietary control could have prompted TCPP due to a premature activation of the HPGA by a toxic effect on the brain. Concerning this latter conjecture, there are currently insufficient data in the literature as to the incidence of TCPP in untreated or insufficiently treated individuals with $\mathrm{PAH}$ deficiency. However, expert opinion corroborated by a poll of the metabolic listserv "metab-l" suggests that this is an extremely rare occurrence. Our patient is the first case where idiopathic TCPP occurred under conditions of perfect dietary control. Since encountering TCPP in our patient we have performed a literature search to identify studies that describe the growth pattern of patients with PKU, but none of them focused on the timing of pubertal onset. Recent studies of larger cohorts of individuals on PKU diets have identified an increased prevalence of obesity and overweight towards adolescence in female subjects, with no specific records about precocious or early pubertal progression $[17,18]$. Lack of evidence for different timing of pubertal onset in PKU patients compared with the general population corroborates our observation, and strongly supports the notion of a co-incidental association between PKU and TCPP. We can not entirely exclude, however, that significant anomalies in the onset and tempo of puberty in PKU patients might have been overlooked by previous population studies.

We conclude that TCPP is a rare coincidental event in children with PKU and can occur independently by the persistently high phenylalanine concentrations.

\section{Consent}

Written informed consent was obtained according to the parents of the patient for publication of this Case report and any accompanying images. A copy of the written consent is available for review by the Editor of this journal.

\footnotetext{
Abbreviations

CPP: Central precocious puberty; PKU: Phenylketonuria; TCPP: True central precocious puberty; HPGA: Hypothalamic-pituitary-gonadal axis; PAH: Phenylalanine hydroxylase; Phe: Phenylalanine; Tyr: Tyrosine; HP: hyperphenylalaninemia; LH: Luteinizing hormone; FSH: Follicle stimulating hormone; Cr: Creatinine.
}

Competing interests

The authors declare they have no competing interests. 


\section{Authors' contributions}

$L L$ reviewed the case note and performed the first draft of the manuscript. CG and MD followed the patient from and Endocrine Point of view, while BS from a metabolic point of view. CG supervised the draft of the manuscript and reviewed it with $\mathrm{MD}$ and BS. All authors read and approve the final manuscript.

\section{Acknowledgements}

The authors would like to thank the patient's family.

\section{Author details}

${ }^{1}$ Paediatric Unit, Department of Medical and Surgical Sciences for the Children and Adults, University of Modena \& Reggio Emilia, via del Pozzo n. 71, 41125 Modena, Italy. ${ }^{2}$ Metabolic Unit, Royal Hospital for Sick Children, Dalnair St, Yorkhill, G3 8SJ Glasgow, UK. ${ }^{3}$ Paediatric Endocrinology Unit, Royal Hospital for Sick Children, Dalnair St, Yorkhill, G3 8SJ Glasgow, UK

${ }^{4}$ Dipartimento di Medicina Pediatrica, Bambino Gesù Children's Hospital, Piazza S. Onofiro 4, 00165 Rome, Italy.

Received: 11 November 2013 Accepted: 8 April 2014

Published: 28 April 2014

\section{References}

1. Grumbach MM, Styne DM: Puberty: Ontogeny, neuroendocrinology, physiology, and disorders. In Williams Textbook of Endocrinology. 9th edition. Edited by Wilson JD, Foster DW, Kronenberg HM, Larsen PR. Philadelphia: W.B. Saunders; 1998:1509-1625.

2. González ER: For puberty that comes too soon, new treatment highly effective. JAMA 1982, 248:1149-1151. 1155.

3. Bridges NA, Christopher JA, Hindmarsh PC, Brook CG: Sexual precocity: sex incidence and etiology. Arch Dis Child 1994, 70:116-118.

4. Fahmy JL, Kaminsky CK, Kaufman F, Nelson MD Jr, Parisi MT: The radiological approach to precocious puberty. Br J Radiol 2000, 73:560-567.

5. Raphaelson MI, Stevens JC, Elders J, Comite F, Theodore WH: Familial spastic paraplegia, mental retardation and precocious puberty. Arch Neurol 1983, 40:809-810.

6. Kurosawa K, Kawame H, Okamoto N, Ochiai Y, Akatsuka A, Kobayashi M, Shimohira M, Mizuno S, Wada K, Fukushima Y, Kawawaki H, Yamamoto T, Masuno M, Imaizumi K, Kuroki Y: Epilepsy and neurological findings in 11 individuals with 1p36 deletion syndrome. Brain Dev 2005, 27:378-382.

7. Ito N, Ihara K, Tsutsumi Y, Miyake N, Matsumoto N, Hara T: Hypothalamic pituitary complications in Kabuki syndrome. Pituitary 2013, 16:133-138.

8. Young C, Wang PJ, Tsai WY, Shen YZ: Precocious puberty in a case with probable Angelman syndrome. Brain Dev 1994, 16:249-252.

9. Waisbren SE, Noel K, Fahrbach K, Cella C, Frame D, Dorenbaum A, Levy H: Phenylalanine blood levels and clinical outcomes in phenylketonuria: a systematic literature review and meta-analysis. Mol Genet Metab 2007, 92:63-70.

10. Brenton DP, Pietz J: Adult care in phenylketonuria and hyperphenylalaninaemia: the relevance of neurological abnormalities. Eur J Pediatr 2000, 159(Suppl 2):114-120.

11. Büyükgebiz B, Eroğlu $Y$, Büy ukgebiz A: True precocious puberty associated with phenylketonuria. J Pediatr Endocrinol 1994, 7:361-363.

12. Freeman JV, Cole TJ, Chinn S, Jones PR, White EM, Preece MA: Cross sectional stature and weight reference curves for the UK, 1990. Arch Dis Child 1995, 73:17-24

13. Marshall WA, Tanner JM: Variations in pattern of pubertal changes in girls. Arch Dis Child 1969, 44:291-303.

14. Tanner JM, Whitehouse RH, Marshall WA, Healy MJR, Goldstein H: Assessment of skeletal maturity and prediction of adult height (TW2 method). 2nd edition. London: Academic; 1983.

15. Christ SE: Asbjørn Følling and the discovery of phenylketonuria. $J$ Hist Neurosci 2003, 12:44-54.

16. Woolf LI: Phenylketonuria in Turkey, Ireland and West Scotland. $J$ Inherit Metab Dis 1994, 17:246-247.
17. Belanger-Quintana A, Martínez-Pardo M: Physical development in patients with phenylketonuria on dietary treatment: a retrospective study. Mol Genet Metab 2011, 104:480-484.

18. Burrage LC, McConnell J, Haesler R, O'Riordan MA, Sutton VR, Kerr DS, McCandless SE: High prevalence of overweight and obesity in females with phenylketonuria. Mol Genet Metab 2012, 107:43-48.

doi:10.1186/1472-6823-14-38

Cite this article as: Lucaccioni et al: Central precocious puberty in a 3 year-old girl with Phenylketonuria: a rare association? BMC Endocrine Disorders 2014 14:38

\section{Submit your next manuscript to BioMed Central and take full advantage of:}

- Convenient online submission

- Thorough peer review

- No space constraints or color figure charges

- Immediate publication on acceptance

- Inclusion in PubMed, CAS, Scopus and Google Scholar

- Research which is freely available for redistribution

Submit your manuscript at www.biomedcentral.com/submit 